International Journal of Pure and Applied Mathematics

Volume 84 No. 4 2013, 397-418

ISSN: 1311-8080 (printed version); ISSN: 1314-3395 (on-line version)

url: http://www.ijpam.eu

doi: http://dx.doi.org/10.12732/ijpam.v84i4.10

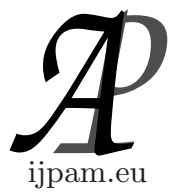

\title{
SHANNON-LIKE PARSEVAL FRAME WAVELETS ON SOME TWO STEP NILPOTENT LIE GROUPS
}

\author{
Vignon Oussa \\ Department of Mathematics \\ Bridgewater State University \\ Bridgewater, MA 02325, USA
}

\begin{abstract}
We construct Shannon-like Parseval frame wavelets on a class of non commutative two-step nilpotent Lie groups. Our work was inspired by a construction given by Azita Mayeli on the Heisenberg group. The tools used here are representation theoretic. However, a great deal of Gabor theory is used for the construction of the wavelets. The construction obtained here is very explicit, and we are even able to compute an upper bound for the $L^{2}$ norm for these Parseval frame wavelets.
\end{abstract}

AMS Subject Classification: 22E25

Key Words: nilpotent, Lie groups, wavelets

\section{Introduction}

A wavelet on $\mathbb{R}^{n}$ is a function generating an orthonormal basis by integral shifts and dilations in $L^{2}\left(\mathbb{R}^{n}\right)$. At this point, a great deal is already known about wavelets on $\mathbb{R}^{n}$. See [8] for example. However, the theory of wavelets on non commutative domains is not as mature. In fact, it is significantly more difficult

Received: January 15, 2013

(c) 2013 Academic Publications, Ltd. url: www.acadpubl.eu 
to construct wavelets over non commutative groups. Several mathematicians have made significant contributions to the field. For example, when it comes to continuous wavelets on locally compact groups, the monograph by Hartmut Führ [9] is a great source of reference. In [4], Currey studies continuous wavelets on nilpotent Lie groups, and Mayeli and Currey developed concepts of wavelet sets on the Heisenberg group in [2]. Also, in [11], wavelets on stratified Lie groups are studied by Lemarié. Other imporant contributions can be found in [10], [12], and [3].

Since the closest objects to $\mathbb{R}^{n}$ are simply connected, connected non commutative nilpotent Lie groups, it is natural to extend the classical results of wavelet theory to this class of groups. Even though topologically, commutative Lie groups, and non commutative simply connected, connected nilpotent Lie groups are identical, their group structures are quite different. Let $N$ be a simply connected, and connected nilpotent Lie group. In order to generate a wavelet system in $L^{2}(N)$, just like it is done in the classical case, we hope to be able to use a set of translations and dilations operators acting on either a single function or a countable set of functions. Naturally, the translation actions should come from the restriction of the left regular representation of the group to some discrete set (or lattice) $\Gamma \subset N$. The dilation actions should be implemented from a discrete subgroup of the outer-automorphism group of $N$. We would like to remark that the existence of a lattice subgroup in $N$ is in fact equivalent to the existence of a rational structure on its Lie algebra [1].

In this paper, we prove the existence and we give an explicit construction of some Shannon-like Parseval frame wavelets on a class of step-two nilpotent Lie groups which we describe as follows. Let $N$ be an $n$-dimensional non commutative, simply connected, connected, nilpotent Lie group with rational structure. We assume that the Lie algebra has a fixed Jordan Hölder-basis,

$$
J=\left\{B_{1}, \cdots, B_{n}\right\}=\left\{\begin{array}{c}
Z_{1}, Z_{2}, \cdots, Z_{n-2 d}, X_{1}, X_{2}, \cdots, X_{d}, \\
Y_{1}, Y_{2}, \cdots, Y_{d}
\end{array}\right\} .
$$

The Lie algebra of $N$ has the following properties: $\mathfrak{n}=\mathfrak{z}(\mathfrak{n}) \oplus \mathfrak{a} \oplus \mathfrak{b}$, where $\mathfrak{z}(\mathfrak{n})=\mathbb{R}$-span $\left\{Z_{1}, Z_{2}, \cdots, Z_{n-2 d}\right\}$ is the center of $\mathfrak{n}$,

$$
\mathfrak{b}=\mathbb{R}-\operatorname{span}\left\{X_{1}, X_{2}, \cdots, X_{d}\right\}
$$

$\mathfrak{a}=\mathbb{R}$-span $\left\{Y_{1}, Y_{2}, \cdots, Y_{d}\right\}$, and $[\mathfrak{a}, \mathfrak{b}] \subseteq \mathfrak{z}(\mathfrak{n})$. Thus, $N$ is isomorphic to a semidirect product group of the type $\left(\mathbb{R}^{n-2 d} \times \mathbb{R}^{d}\right) \rtimes \mathbb{R}^{d}$. Moreover $\exp (\mathfrak{z}(\mathfrak{n}) \oplus \mathfrak{a})$ is a maximal commutative normal subgroup of $N$, and of course, $\mathfrak{z}(\mathfrak{n}) \oplus \mathfrak{a}$ is a maximal commutative ideal of $\mathfrak{n}$. We also assume that the generic rank of the 
matrix

$$
\left(\begin{array}{ccc}
{\left[B_{1}, B_{1}\right]} & \cdots & {\left[B_{1}, B_{n}\right]} \\
\vdots & \ddots & \vdots \\
{\left[B_{n}, B_{1}\right]} & \cdots & {\left[B_{n}, B_{n}\right]}
\end{array}\right)
$$

is equal to $2 d$ on $[\mathfrak{n}, \mathfrak{n}]$.This class of groups which contains the Heisenberg groups, direct products of Heisenberg groups with $\mathbb{R}^{m}$, and various generalizations of the Heisenberg groups, has also been studied in [13].

Let $L$ be the left regular representation of $N$ acting in $L^{2}(N)$ endowed with its canonical left Haar measure. We show that there exists a group

$$
H=\left\{A^{j}: A=\exp (U), j \in \mathbb{Z}\right\}<\operatorname{Aut}(N),
$$

such that $\left[U, Z_{i}\right]=\ln (2) Z_{i}$, for $1 \leq i \leq n-2 d,\left[U, Y_{k}\right]=\ln (2) Y_{k}$, for $1 \leq k \leq d$, and $\left[U, X_{k}\right]=0$, for $1 \leq k \leq d$. In other words, $H$ is isomorphic to a discrete subgroup of the automorphism group of $N$. Defining a unitary representation of $H$ acting by non-expansive dilations on $L^{2}(N)$, such that $D_{A}: L^{2}(N) \rightarrow$ $L^{2}(N)$,

$$
f(\cdot) \mapsto \operatorname{det}\left(A d_{A}\right)^{-1 / 2} f\left(A^{-1} \cdot\right),
$$

our main results are summarized in the following terms. We show the existence of a discrete set

$$
\Gamma=\prod_{k=1}^{n-2 d} \exp \left(\mathbb{Z} Z_{k}\right) \prod_{k=1}^{d} \exp \left(\mathbb{Z} Y_{k}\right) \prod_{k=1}^{d} \exp \left(\mathbb{Z} X_{k}\right) \subset N,
$$

and an infinite countable family of functions $\left\{f_{k}: k \in \mathbb{I}\right\} \subset L^{2}(N)$ such that the system

$$
\left\{D_{A^{j}} L(\gamma) f_{k}: \gamma \in \Gamma, j \in \mathbb{Z}, k \in \mathbb{I}\right\}
$$

is a Parseval frame in $L^{2}(N)$. For a class of finite-multiplicity subspaces of $L^{2}(N)$ there exists a finite number of functions

$$
\left\{f_{k}: k \in \mathbb{A}\right\} \subset L^{2}(N)
$$

such that the system

$$
\left\{D_{A^{j}} L(\gamma) f_{k}: \gamma \in \Gamma, j \in \mathbb{Z}, k \in \mathbb{A}\right\}
$$

forms a Parseval frame in $L^{2}(N)$. In particular for multiplicity-free subspaces, the set $\mathbb{A}$ is a singleton, and in all cases, $\left\|f_{k}\right\|_{L^{2}(N)} \leq 2^{\frac{2 d-n}{2}}$.

We organize the paper as follows. In the second and third section, we review some important notions of Gabor theory, and analysis on nilpotent Lie groups. The main result is proved in the fourth section, and a construction of Parseval frame wavelets is also given in the same section. 


\section{Preliminaries}

A lattice $\Lambda$ in $\mathbb{R}^{2 d}$ is a discrete subgroup of the additive group $\mathbb{R}^{2 d}$ that is $\Lambda=M \mathbb{Z}^{2 d}$ with $M$ being a non singular matrix. A separable lattice has the form $\Lambda=A \mathbb{Z}^{d} \times B \mathbb{Z}^{d}$. The volume of a lattice $\Lambda=M \mathbb{Z}^{d}$ equals the Lebesgue measure of $\mathbb{R}^{d} / \Lambda$ that is $\operatorname{vol}\left(\mathbb{R}^{d} / \Lambda\right)=|\operatorname{det} M|$ and the density of $\Lambda$ is $d(\Lambda)=(\operatorname{vol}(\Lambda))^{-1}$. Let $A \mathbb{Z}^{d} \times B \mathbb{Z}^{d}$ be a separable lattice in $\mathbb{R}^{2 d}$, and $g \in L^{2}\left(\mathbb{R}^{d}\right)$. A Gabor system $\mathcal{G}\left(g, A \mathbb{Z}^{d} \times B \mathbb{Z}^{d}\right)$ is a sequence of functions defined as

$$
\left\{e^{2 \pi i\langle t, y\rangle} g(t-x): y \in B \mathbb{Z}^{d}, x \in A \mathbb{Z}^{d}\right\} .
$$

For example, $\mathcal{G}\left(\chi_{[0,1)}, \mathbb{Z}^{d} \times \mathbb{Z}^{d}\right)$ is a Gabor system, and an orthonormal basis in $L^{2}\left(\mathbb{R}^{d}\right)$. A sequence $\left\{f_{n}: n \in \mathbb{Z}\right\}$ of elements in a Hilbert space $H$ is called a frame if there are constant $A, B>0$ such that

$$
A\|f\|^{2} \leq \sum_{n \in \mathbb{Z}}\left|\left\langle f, f_{n}\right\rangle\right|^{2} \leq B\|f\|^{2} \text { for all } f \in H .
$$

The numbers $A, B$ in the definition of a frame are called lower and upper bounds respectively. A frame is a tight frame if $A=B$ and a normalized tight frame or Parseval frame if $A=B=1$.

The following results are well-known in Gabor theory, and can be found in [7].

Proposition 1. Let $\Lambda$ be a full rank lattice in $\mathbb{R}^{2 d}$ with $d(\Lambda) \geq 1$. The following are equivalent

1. There exists $g \in L^{2}\left(\mathbb{R}^{d}\right)$ such that $\mathcal{G}(g, \Lambda)$ is complete in $L^{2}\left(\mathbb{R}^{d}\right)$.

2. There exists $g \in L^{2}\left(\mathbb{R}^{d}\right)$ such that $\mathcal{G}(g, \Lambda)$ is a frame in $L^{2}\left(\mathbb{R}^{d}\right)$.

3. There exists $g \in L^{2}\left(\mathbb{R}^{d}\right)$ such that $\mathcal{G}(g, \Lambda)$ is a Parseval frame in $L^{2}\left(\mathbb{R}^{d}\right)$.

Proposition 2. Let $\mathcal{G}\left(g, A \mathbb{Z}^{d} \times B \mathbb{Z}^{d}\right)$ be a Gabor system. If

$$
\mathcal{G}\left(g, A \mathbb{Z}^{d} \times B \mathbb{Z}^{d}\right)
$$

is a Parseval frame in $L^{2}\left(\mathbb{R}^{d}\right)$ then $\|g\|^{2}=|\operatorname{det} A \operatorname{det} B|$.

Proof. See proof of Theorem 1.3 in [7]. 
Let $\psi \in L^{2}(\mathbb{R})$. In the classical sense, we say $\psi$ is a wavelet iff the system

$$
\left\{\psi_{j k}(x)=2^{j / 2} \psi\left(2^{j} x-k\right): j, k \in \mathbb{Z}\right\}
$$

forms an orthonormal basis in $L^{2}(\mathbb{R})$, and we say $\psi$ is a (Parseval) frame wavelet iff the system (1) forms a (Parseval) frame in $L^{2}(\mathbb{R})$. There are several ways to construct wavelets in $L^{2}(\mathbb{R})$. For example the concept of wavelet sets is exposed in [6]. Also, the oldest known wavelet is the Haar wavelet, given by

$$
\psi(x)=\left\{\begin{array}{c}
1 \text { if } x \in[0,1 / 2) \\
-1 \text { if } x \in[1 / 2,1) \\
0 \text { if } x \in \mathbb{R} \backslash(0,1)
\end{array} .\right.
$$

The Haar wavelet has been discovered in 1910 way before the concepts of wavelets were developed. Another well-known example is the function $\psi$ whose Fourier transform is the characteristic function of the Littlewood-Paley wavelet set $[-2 \pi,-\pi) \cup[\pi, 2 \pi)$, and

$$
\psi(x)=\frac{\sin (2 \pi x)-\sin (\pi x)}{\pi x}
$$

is a wavelet in $L^{2}(\mathbb{R})$. The Littlewood-Paley wavelet set will be of special interest in this paper.

\section{Analysis on Nilpotent Lie Groups}

The unitary dual of a simply connected, connected nilpotent Lie group is wellunderstood via the orbit method [1]. The orbit method is simply stated in the following terms. Up to isomorphism, the unitary irreducible representations of any simply connected, connected nilpotent Lie group are in a one-to-one correspondence with the coadjoint orbits of the Lie group on elements of the dual of its Lie algebra. In other words, if two linear functionals belong to the same orbit, their corresponding unitary irreducible representations must be isomorphic. This correspondence is known as Kirillov's map. If $N$ is a nilpotent Lie group, the Fourier transform just like on Euclidean spaces is really defined on $L^{1}(N) \cap L^{2}(N)$ as

$$
\mathcal{F} f(\lambda)=\int_{N} f(n) \pi_{\lambda}(n) d n
$$

where $\left(\pi_{\lambda}, \mathcal{H}_{\lambda}\right)$ is an irreducible representation corresponding to the linear functional $\lambda$ via Kirillov's map. Clearly, the Fourier transform, as given above is 
weakly defined and should be understood as follows. Let $u, v$ be 2 arbitrary vectors in the Hilbert space on which we realized the action of $\pi_{\lambda}$. We have

$$
\langle\mathcal{F} f(\lambda) u, v\rangle=\int_{N} f(n)\left\langle\pi_{\lambda}(n) u, v\right\rangle d n .
$$

Since $L^{1}(N) \cap L^{2}(N)$ is dense in $L^{2}(N)$, the extension of the Fourier transform on $L^{2}(N)$ is naturally called the Plancherel transform, which we will denote in this paper by $\mathcal{P}$. $\mathcal{P}$ induces an isometry on $L^{2}(N)$, and if $\Sigma$ is a parametrizing set for the unitary dual of $N$, there exists a measure called the Plancherel measure such that

$$
\mathcal{P}\left(L^{2}(N)\right)=\int_{\Sigma}^{\oplus}\left(\mathcal{H}_{\lambda} \otimes \mathcal{H}_{\lambda}\right) d \mu(\lambda) .
$$

Moreover, letting $\left(L, L^{2}(N)\right)$ be the left regular representation of $N$,

$$
\mathcal{P} \circ L \circ \mathcal{P}^{-1}=\int_{\Sigma}^{\oplus}\left(\pi_{\lambda} \otimes 1_{\mathcal{H}_{\lambda}}\right) d \mu(\lambda),
$$

where $1_{\mathcal{H}_{\lambda}}$ is the identity operator defined on $\mathcal{H}_{\lambda}$, we refer the interested reader to [1] which is a standard reference book for the representation theory of nilpotent Lie groups.

Definition 3. Let $\mathfrak{g}$ be an $n$-dimensional Lie algebra over the reals. We say that $\mathfrak{g}$ has a rational structure if and only if there exists an $\mathbb{R}$-basis $\left\{V_{1}, V_{2}, \cdots, V_{n}\right\}$ for $\mathfrak{g}$ having rational structure constants, and $\mathfrak{g}_{\mathbb{Q}}=\mathbb{Q}-\operatorname{span}\left\{V_{1}\right.$, $\left.V_{2}, \cdots, V_{n}\right\}$ provides a rational structure such that $\mathfrak{g} \cong \mathfrak{g}_{\mathbb{Q}} \otimes \mathbb{R}$.

Definition 4. Let $G$ be a nilpotent Lie group. A lattice subgroup is a uniform subgroup $\Gamma$ of $G$ such that $\Lambda=\log \Gamma$ is an additive subgroup of $\mathfrak{g}$.

Definition 5. Let $G$ be a second-countable, unimodular locally compact group. An irreducible representation $\pi$ of $G$ acting in $H_{\pi}$ is said to be squareintegrable if for every $u, v \in H_{\pi}$ the matrix coefficient function $x \mapsto\langle\pi(x) u, v\rangle$ is in $L^{2}(G)$.

Square-integrable modulo the center nilpotent Lie groups are rather appealing compared to other types of nilpotent Lie groups because their unitary duals admit simpler descriptions, and they are essentially identified with Zariski open sets of Euclidean spaces.

Let $N$ be a simply connected, connected, non commutative nilpotent Lie group with Lie algebra $\mathfrak{n}$ of dimension $n$ over $\mathbb{R}$, with a rational structure. We start by fixing an ordered Jordan Hölder basis

$$
J=\left\{B_{1}, B_{2}, \cdots, B_{n}\right\}=\left\{Z_{1}, Z_{2}, \cdots, Z_{n-2 d}, Y_{1}, \cdots, Y_{d}, X_{1}, \cdots, X_{d}\right\},
$$


such that

C1. $\mathfrak{n}=\mathfrak{z}(\mathfrak{n}) \oplus \mathfrak{a} \oplus \mathfrak{b}$ where $\mathbb{R}-\operatorname{span}\left\{Z_{1}, Z_{2}, \cdots, Z_{n-2 d}\right\}=\mathfrak{z}(\mathfrak{n}), \mathbb{R}-\operatorname{span}\left\{Y_{1}\right.$, $\left.Y_{2}, \cdots, Y_{d}\right\}=\mathfrak{a}$, and $\mathbb{R}-\operatorname{span}\left\{X_{1}, X_{2}, \cdots, X_{d}\right\}=\mathfrak{b}$.

C2. $\mathfrak{z}(\mathfrak{n}) \oplus \mathfrak{a}$ is a commutative ideal of $\mathfrak{n}$, and $\mathfrak{b}$ is a commutative subalgebra (not an ideal) of $\mathfrak{n}$ such that $[\mathfrak{a}, \mathfrak{b}] \subseteq \mathfrak{z}(\mathfrak{n})$.

C3. Defining the matrix $M(J)$ of structure constants related to $J$ such that $M(J)_{i, j}=\left[B_{i}, B_{j}\right]$, and letting $\mathbf{0}_{p \times p}$ be the zero matrix of order $p$, we assume that

$$
M(J)=\left(\begin{array}{ccc}
\mathbf{0}_{n-2 d \times n-2 d} & \mathbf{0}_{n-2 d \times d} & \mathbf{0}_{n-2 d \times d} \\
\mathbf{0}_{d \times n-2 d} & \mathbf{0}_{d \times d} & -V \\
\mathbf{0}_{d \times n-2 d} & V & \mathbf{0}_{d \times d}
\end{array}\right)^{\prime}
$$

and we define the matrix

$$
V=\left(\begin{array}{ccc}
{\left[X_{1}, Y_{1}\right]} & \cdots & {\left[X_{1}, Y_{d}\right]} \\
\vdots & \ddots & \vdots \\
{\left[X_{d}, Y_{1}\right]} & \cdots & {\left[X_{d}, Y_{d}\right]}
\end{array}\right)
$$

such that $\operatorname{det}(V)$ is a nonzero polynomial with rational coefficients defined over $[\mathfrak{n}, \mathfrak{n}]$.

Let $\mathfrak{n}^{*}$ the dual vector space of $\mathfrak{n}$. The coadjoint action of $N$ on $\mathfrak{n}^{*}$ is denoted multiplicatively such that for any given $\exp W \in N$ and $\lambda \in \mathfrak{n}^{*}$

$$
\exp W \cdot \lambda\left(\sum_{k=1}^{n} u_{k} U_{k}\right)=\lambda\left(e^{a d_{-W}}\left(\sum_{k=1}^{n} u_{k} U_{k}\right)\right) .
$$

Lemma 6. If a Lie group $N$ satisfies conditions $C 1, C 2$, and $C 3$, then $N$ is a step two, square-integrable modulo the center nilpotent Lie group. Moreover, for any element $\lambda \in \mathfrak{n}^{*}$ we define the matrix

$$
B(\lambda)=\left(\begin{array}{ccc}
\lambda\left[X_{1}, Y_{1}\right] & \cdots & \lambda\left[X_{1}, Y_{d}\right] \\
\vdots & \ddots & \vdots \\
\lambda\left[X_{d}, Y_{1}\right] & \cdots & \lambda\left[X_{d}, Y_{d}\right]
\end{array}\right)
$$

such that $\operatorname{det} B(\lambda)$ is a nonzero polynomial defined over $[\mathfrak{n}, \mathfrak{n}]^{*}$. The dual of $N$ denoted $\widehat{N}$ is up to a null set parametrized by the manifold

$$
\Sigma=\left\{\lambda \in \mathfrak{n}^{*}: \operatorname{det} B(\lambda) \neq 0, \lambda\left(X_{i}\right)=\lambda\left(Y_{i}\right)=0,1 \leq i, j \leq d\right\},
$$


which is a Zariski open subset of $\mathfrak{z}(\mathfrak{n})^{*}$. Also, the Plancherel measure (associated to our fixed Jordan-Hölder basis) is given by $d \mu(\lambda)=|\operatorname{det} B(\lambda)| d \lambda$ where $d \lambda$ is the canonical Lebesgue measure defined on $\Sigma$.

Proof. Clearly, $N$ is a step-two nilpotent Lie group because, it is noncommutative and $[\mathfrak{n}, \mathfrak{n}] \subseteq \mathfrak{z}(\mathfrak{n})$. Next, $N$ being a nilpotent Lie group, according to the orbit method, its unitary dual is in one-to-one correspondence with the coadjoint orbits of $N$ in $\mathfrak{n}^{*}$. An algorithm for the computation of a smooth cross-section parameterizing (up to a null set) almost all of the irreducible representations is available in Chapter 3 of [1]. Furthermore, a formula for the computation of the Plancherel measure is also available in [1] (Chapter 4). To show that $N$ is square-integrable modulo the center, according to 5.4.4 Corollary in [1], it suffices to show that the null-space of the matrix $\left(\lambda\left[B_{i}, B_{j}\right]\right)_{1 \leq i, j \leq n}$ is equal to the central ideal $\mathfrak{z}(\mathfrak{n})$. This is clearly true since by assumption,

$$
\operatorname{rank}\left(\lambda\left[B_{i}, B_{j}\right]\right)_{1 \leq i, j \leq n}=\operatorname{rank}(M(J))=2 d,
$$

and the first $n-2 d$ columns of the matrix $\left(\lambda\left[B_{i}, B_{j}\right]\right)_{1 \leq i, j \leq n}$ are all zeros (while the remaining $2 d$ columns are linearly independent).

From now on, we may just assume that $N$ is a simply connected, connected nilpotent Lie group endowed with a rational structure satisfying conditions $\mathrm{C} 1$, $\mathrm{C} 2$ and $\mathrm{C} 3$ as defined previously.

Lemma 7. For a fixed linear functional $\lambda \in \Sigma$ (see 4), a corresponding irreducible representation of $N$ is denoted $\pi_{\lambda}$ and is realized as acting in $L^{2}\left(\mathbb{R}^{d}\right)$ such that for $\phi \in L^{2}\left(\mathbb{R}^{d}\right)$,

$$
\begin{aligned}
\pi_{\lambda}\left(\exp \left(z_{1} Z_{1}+\cdots+z_{n-2 d} Z_{n-2 d}\right)\right) \phi(t) & =\exp (2 \pi i\langle\lambda, z\rangle) \phi(t), \\
\pi_{\lambda}\left(\exp \left(y_{1} Y_{1}+\cdots+y_{d} Y_{d}\right)\right) \phi(t) & =\exp (2 \pi i\langle t, B(\lambda) y\rangle) \phi(t), \\
\pi_{\lambda}\left(\exp x_{1} X_{1}+\cdots+x_{d} X_{d}\right) \phi(t) & =\phi(t-x),
\end{aligned}
$$

where $z=\left(z_{1}, \cdots, z_{n-2 d}\right) \in \mathbb{R}^{n-2 d}, y=\left(y_{1}, \cdots, y_{d}\right) \in \mathbb{R}^{d}, x=\left(x_{1}, \cdots, x_{d}\right) \in$ $\mathbb{R}^{d}$.

For a proof of the lemma, we invite the reader to refer to Chapter 2 in [1] for general nilpotent Lie groups, or [13] for the class of groups considered in this paper.

Remark 8. From our definition of Gabor systems, for $\phi \in L^{2}\left(\mathbb{R}^{d}\right)$,

$$
\pi_{\lambda}\left(\exp \left(\mathbb{Z} Y_{1}+\cdots+\mathbb{Z} Y_{d}\right) \exp \left(\mathbb{Z} X_{1}+\cdots+\mathbb{Z} X_{d}\right)\right) \phi
$$

is a Gabor system in $L^{2}\left(\mathbb{R}^{d}\right)$ of the type $G\left(\phi, \mathbb{Z}^{d} \times B(\lambda) \mathbb{Z}^{d}\right)$. 
Let $\exp U \in \operatorname{Aut}(N)$ such that $U$ is a derivation of the Lie algebra of $N$. For all $i$ such that $1 \leq i \leq n$ and for any real number $\alpha$, the following must hold

1. $\left[U, \alpha B_{i}\right]=\alpha B_{i}$,

2. $\left[U, B_{i}+B_{j}\right]=\left[U, B_{i}\right]+\left[U, B_{j}\right]$,

3. $\left[U, B_{i}\right]=-\left[B_{i}, U\right]$,

4. (Jacobi identity) $\left[\left[U, B_{i}\right], B_{j}\right]+\left[\left[B_{i}, B_{j}\right], U\right]+\left[\left[B_{j}, U\right], B_{i}\right]=0$.

Lemma 9. Let $\exp U \in \operatorname{Aut}(N)$ such that $\left[U, X_{i}\right]=a X_{i}$ and $\left[U, Y_{i}\right]=b Y_{i}$ for some $a, b \in \mathbb{R}$ and for all $i$ such that $1 \leq i \leq d$. There exists a matrix representation of the linear adjoint action of $\mathbb{R} U$ in $\mathfrak{g l}(\mathfrak{n})$ such that

$$
\begin{aligned}
& a d: \mathbb{R} U \rightarrow \operatorname{Diag}(\mathfrak{n}) \subset \mathfrak{g l}(\mathfrak{n}) \\
& U \mapsto\left(\begin{array}{ccc}
(a+b) \mathbf{I}_{n-2 d} & \cdots & \mathbf{0} \\
\vdots & b \mathbf{I}_{d} & \vdots \\
\mathbf{0} & \cdots & a \mathbf{I}_{d}
\end{array}\right),
\end{aligned}
$$

where $\mathbf{I}_{q}$ represents the identity matrix of order $q$.

Proof. To prove the Lemma, it suffices to check axioms 1,2,3, and 4. Clearly, axioms 1,2 , and 3 are satisfied. It remains to prove that the Jacobi identity is satisfied as well. If $\left[U, X_{i}\right]=a X_{i}$ and $\left[U, Y_{i}\right]=b Y_{i}$, by the Jacobi identity, $\left[\left[X_{i}, Y_{j}\right], U\right]+\left[\left[Y_{j}, U\right], X_{i}\right]+\left[\left[U, X_{i}\right], Y_{j}\right]=0 \Rightarrow\left[U,\left[X_{i}, Y_{j}\right]\right]=(a+b)\left[X_{i}, Y_{j}\right]$. For central elements, there are two separate cases to consider. First, we suppose that $Z_{i} \in J$ is an element of the commutator ideal $[\mathfrak{n}, \mathfrak{n}] \leq \mathfrak{z}(\mathfrak{n})$. That is $Z=\sum_{1 \leq i, j \leq d} \alpha_{i, j}\left[X_{i}, Y_{j}\right]$. So, $[U, Z]=\sum_{1 \leq i, j \leq d} \alpha_{i, j}\left[U,\left[X_{i}, Y_{j}\right]\right]=$ $(a+b) \sum_{1 \leq i, j \leq d} \alpha_{i, j}\left[\bar{X}_{i}, Y_{j}\right]=(a+b) Z$. Now assume that

$$
Z=\sum_{1 \leq i, j \leq d} \alpha_{i, j}\left[X_{i}, Y_{j}\right]+W
$$

such that $W$ is a central element but $W \notin[\mathfrak{n}, \mathfrak{n}]$. Defining $[U, W]=(a+b) W$ does not violate any of the axioms required for $U$ to induce a linear adjoint action on the Lie algebra $\mathfrak{n}$. Thus, there exits $U$ such that $[U, Z]=(a+b) Z$. This completes the proof. 
Corollary 10. Let $\exp U \in \operatorname{Aut}(N)$ such that $\left[U, X_{i}\right]=0$ and $\left[U, Y_{i}\right]=$ $\ln (2) Y_{i}$ for all $i$ such that $1 \leq i \leq d$. There exists a matrix representation of the linear adjoint action of $\mathbb{R} U$ in $\mathfrak{g l}(\mathfrak{n})$ such that

$$
\begin{aligned}
& a d_{U}=\left(\begin{array}{ccc}
\ln (2) \mathbf{I}_{n-2 d} & \cdots & \mathbf{0} \\
\vdots & \ln (2) \mathbf{I}_{d} & \vdots \\
\mathbf{0} & \cdots & \mathbf{0}_{d}
\end{array}\right) \text {, and } \\
& A d_{\exp U}=\left(\begin{array}{ccc}
2 \mathbf{I}_{n-2 d} & \cdots & \mathbf{0} \\
\vdots & 2 \mathbf{I}_{d} & \vdots \\
\mathbf{0} & \cdots & \mathbf{I}_{d}
\end{array}\right)
\end{aligned}
$$

where $a d_{U}$ is the derivative of $A d_{\exp U}$.

Proof. For the existence of

$$
a d_{U}=\left(\begin{array}{ccc}
\ln (2) \mathbf{I}_{n-2 d} & \cdots & \mathbf{0} \\
\vdots & \ln (2) \mathbf{I}_{d} & \vdots \\
\mathbf{0} & \cdots & \mathbf{0}_{d}
\end{array}\right)
$$

we use Lemma 9. Next, since $A d_{\exp U}=\exp a d_{U}$, we have

$$
A d_{\exp U}=\exp \left(\begin{array}{ccc}
\ln (2) \mathbf{I}_{n-2 d} & \cdots & \mathbf{0} \\
\vdots & \ln (2) \mathbf{I}_{d} & \vdots \\
\mathbf{0} & \cdots & \mathbf{0}_{d}
\end{array}\right)=\left(\begin{array}{ccc}
2 \mathbf{I}_{n-2 d} & \cdots & \mathbf{0} \\
\vdots & 2 \mathbf{I}_{d} & \vdots \\
\mathbf{0} & \cdots & \mathbf{I}_{d}
\end{array}\right) .
$$

Proposition 11. Let $\mathbf{I}_{m}$ be the $d \times d$ identity matrix. Let $\phi: \mathbb{R}^{m} \rightarrow \mathbb{R}$ be a homogeneous polynomial and let $\rho$ be the Lebesgue measure defined on $\mathbb{R}^{m}$. There exits a measurable set $E \subset \mathbb{R}^{m}$ such that the collection of sets $\left\{\left(2 \mathbf{I}_{m}\right)^{j} E: j \in \mathbb{Z}\right\}$ satisfies the following.

1. $\rho\left(\left(2 \mathbf{I}_{m}\right)^{j} E \cap\left(2 \mathbf{I}_{m}\right)^{j^{\prime}} E\right)=0$ for any $j, j^{\prime} \in \mathbb{Z}$, and $j \neq j^{\prime}$.

2. $\rho\left(\mathbb{R}^{m}-\cup_{j \in \mathbb{Z}}\left(2 \mathbf{I}_{m}\right)^{j} E\right)=0$.

3. $E \subseteq \phi^{-1}([-1,1])$. 
Proof. First, notice that (1), and (2) together is equivalent to the fact that the collection of sets $\left\{\left(2 \mathbf{I}_{m}\right)^{j} E: j \in \mathbb{Z}\right\}$ forms a measurable partition of $\mathbb{R}^{m}$. Let $S=[-1 / 2,1 / 2]^{m} \backslash[-1 / 4,1 / 4]^{m} \subset \mathbb{R}^{m}$. Clearly $S$ satisfies conditions (1) and (2). If $S \subseteq \phi^{-1}([-1,1])$ then, we are done. Now assume that $S \nsubseteq$ $\phi^{-1}[-1,1] . \phi$ being a continuous map, there exists $\epsilon>0$ such that $\phi^{-1}[-1,1] \supset$ $(-\epsilon, \epsilon)^{m}$. We pick $j=j(\epsilon) \in \mathbb{Z}$ such that $\left(2 \mathbf{I}_{m}\right)^{j(\epsilon)} S \subset(-\epsilon, \epsilon)^{m}$, and we let $E=\left(2 \mathbf{I}_{m}\right)^{j(\epsilon)} S$. It is now clear that $E$ satisfies conditions (1),(2), and (3), and the proposition is proved.

Remark 12. From now on, we identify the spectrum, $\Sigma(4)$ with an open subset of $\mathbb{R}^{n-2 d} \cong \mathfrak{z}(\mathfrak{n})^{*}$. Thus, we abuse the notation when we make the following statement:

$$
\Sigma=\left\{\left(\lambda_{1}, \cdots, \lambda_{n-2 d}, 0, \cdots, 0\right): \lambda_{i} \in \mathbb{R}\right\} \subset \mathbb{R}^{n-2 d}
$$

It should be understood that, we are assuming that the correct identification is made for $\Sigma$ by suppressing all of the zero coordinates. Otherwise, (7) makes no sense of course.

Now, we would like to specialize Prop 11 to the class of nilpotent Lie groups considered in this paper.

Lemma 13. Identifying $\Sigma$ with a Zariski open subset of $\mathbb{R}^{n-2 d} \equiv \mathfrak{z}(\mathfrak{n})^{*}$, there exists a Jordan-Holder basis for the Lie algebra $\mathfrak{n}$ of $N$ such that for

$$
\lambda \in\left([-1 / 2,1 / 2]^{n-2 d} \backslash[-1 / 4,1 / 4]^{n-2 d}\right) \cap \Sigma,
$$

we have $|\operatorname{det} B(\lambda)| \leq 1$.

Proof. Referring to (3) we recall that

$$
B(\lambda)=\left(\begin{array}{ccc}
\lambda\left[X_{1}, Y_{1}\right] & \cdots & \lambda\left[X_{1}, Y_{d}\right] \\
\vdots & \ddots & \vdots \\
\lambda\left[X_{d}, Y_{1}\right] & \cdots & \lambda\left[X_{d}, Y_{d}\right]
\end{array}\right)
$$

Let $\mathbf{r}$ be a homogeneous polynomial over $\Sigma$ such that $\mathbf{r}: \Sigma \rightarrow \mathbb{R}, \lambda \mapsto \operatorname{det} B(\lambda)$. From Lemma 11, there exits a measurable set $E \subset \Sigma$ (identified with a conull subset of $\mathbb{R}^{n-2 d}$ ) such that the collection of sets $\left\{2 \mathbf{I}_{m}^{j}(E): j \in \mathbb{Z}\right\}$ satisfies all of the conditions stated in Lemma 11. If

$$
\left([-1 / 2,1 / 2]^{n-2 d} \backslash[-1 / 4,1 / 4]^{n-2 d}\right) \cap \Sigma \subset \mathbf{r}^{-1}[-1,1],
$$


we are done. Otherwise, it not too hard to see that we can modify at least one element of the Jordan-Hölder basis

$$
J=\left\{Z_{1}, Z_{2}, \cdots, Z_{n-2 d}, Y_{1}, \cdots, Y_{d}, X_{1}, \cdots, X_{d}\right\}
$$

to satisfy equation 8 . In fact, let us assume that

$$
\left([-1 / 2,1 / 2]^{n-2 d} \backslash[-1 / 4,1 / 4]^{n-2 d}\right) \cap \Sigma
$$

is not contained in $\mathbf{r}^{-1}[-1,1]$. Clearly,

$$
[-1 / 2,1 / 2]^{n-2 d} \cap \Sigma \cap \mathbf{r}^{-1}[-1,1]
$$

is not empty. Let $\frac{1}{k} \in \mathbb{Q}$, and replace $X_{1}$ with $\frac{1}{k} X_{1}$, such that $\mathbf{r}_{k}(\lambda)=\operatorname{det} B_{k}(\lambda)$ where

$$
\operatorname{det} B_{k}(\lambda)=\operatorname{det}\left(\begin{array}{ccc}
\frac{1}{k} \lambda\left(\left[X_{1}, Y_{1}\right]\right) & \cdots & \frac{1}{k} \lambda\left(\left[X_{1}, Y_{d}\right]\right) \\
\vdots & \ddots & \vdots \\
\lambda\left(\left[X_{d}, Y_{1}\right]\right) & \cdots & \lambda\left(\left[X_{d}, Y_{d}\right]\right)
\end{array}\right)=\frac{1}{k} \operatorname{det} B(\lambda) .
$$

As $k \rightarrow \infty, \mathbf{r}_{k}^{-1}[-1,1] \rightarrow \Sigma$. Certainly, since

$$
[-1 / 2,1 / 2]^{n-2 d} \backslash[-1 / 4,1 / 4]^{n-2 d}
$$

is bounded, for $k$ large enough, we obtain

$$
\left([-1 / 2,1 / 2]^{n-2 d} \backslash[-1 / 4,1 / 4]^{n-2 d}\right) \cap \Sigma \subseteq \mathbf{r}_{k}^{-1}([-1,1]) .
$$

Finally, replacing

$$
J=\left\{Z_{1}, Z_{2}, \cdots, Z_{n-2 d}, Y_{1}, \cdots, Y_{d}, X_{1}, \cdots, X_{d}\right\}
$$

with $\left\{Z_{1}, Z_{2}, \cdots, Z_{n-2 d}, Y_{1}, \cdots, Y_{d}, \frac{1}{k} X_{1}, \cdots, X_{d}\right\}$, we complete the proof.

Now, we make a choice of coordinates which will be convenient for our purpose throughout this paper. Let $m \in N$ and $\exp U \in \operatorname{Aut}(N)$. We write the automorphic action induced by $A$ as follows $A d_{A}(m)=A(m)$. Given

$$
m=\exp \left(\sum_{l=1}^{n-2 d} z_{l} Z_{l}\right) \exp \left(\sum_{k=1}^{d} y_{k} Y_{k}\right) \exp \left(\sum_{k=1}^{d} x_{k} X_{k}\right)
$$

which we identify with the vector $\left(z_{1}, \cdots, z_{n-2 d}, y_{1}, \cdots, y_{d}, x_{1}, \cdots, x_{d}\right)$, it is easy to see that

$$
A(m)=\exp \left(\sum_{l=1}^{n-2 d} 2 z_{l} Z_{l}\right) \exp \left(\sum_{k=1}^{d} 2 y_{k} Y_{k}\right) \exp \left(\sum_{k=1}^{d} x_{k} X_{k}\right) .
$$


Remark 14. Identifying $\Sigma$ with a subset of $\mathbb{R}^{n-2 d}$, let $A=\exp U$, and $\lambda \in \Sigma$. The coadjoint action of $A$ on $\lambda$ is computed as follows

$$
A \cdot \lambda=\exp U \cdot\left(\lambda_{1}, \cdots, \lambda_{n-2 d}\right)=\frac{1}{2} \mathbf{I}_{n-2 d}\left(\lambda_{1}, \cdots, \lambda_{n-2 d}\right) .
$$

Definition 15. We define

$$
\begin{aligned}
\Gamma_{1} & =\exp \left(\sum_{l=1}^{n-2 d} \mathbb{Z} Z_{l}\right) \in \mathfrak{z}(\mathfrak{n}), \Gamma_{2}=\exp \left(\sum_{i=1}^{d} \mathbb{Z} Y_{i}\right), \\
\text { and } \Gamma_{3} & =\exp \left(\sum_{k=1}^{d} \mathbb{Z} X_{k}\right)
\end{aligned}
$$

such that $\Gamma=\Gamma_{1} \Gamma_{2} \Gamma_{3}$ and

$$
\Gamma=\exp \left(\sum_{l=1}^{n-2 d} \mathbb{Z} Z_{l}\right) \exp \left(\sum_{i=1}^{d} \mathbb{Z} Y_{i}\right) \exp \left(\sum_{k=1}^{d} \mathbb{Z} X_{k}\right) .
$$

Lemma 16. The group generated by the set $\Gamma$ as defined in (10) is a lattice subgroup of $N$.

The proof is elementary. Thus we will omit it. The interested reader is referred to Chapter 5 of the book [1]

\section{Existence and Construction of Wavelets}

In this section, taking advantage of the representation theory of nilpotent Lie groups, we will provide an explicit construction of wavelets over non commutative nilpotent domains. Let $\mu$ denote the Plancherel measure for the group $N$ and $\mathcal{P}$ the Plancherel transform defined on $L^{2}(N)$. From now on, we set

$$
E=\left([-1 / 2,1 / 2]^{n-2 d} \backslash[-1 / 4,1 / 4]^{n-2 d}\right) \cap \Sigma .
$$

Also, recall that via the Fourier transform, given $x \in N$, we have $\mathcal{F}(L(x) f)(\lambda)=$ $\pi_{\lambda}(x) \mathcal{F} f(\lambda)$ and

$$
\mathcal{P}\left(L^{2}(N)\right)=\int_{\Sigma}^{\oplus} L^{2}\left(\mathbb{R}^{d}\right) \otimes L^{2}\left(\mathbb{R}^{d}\right)|\operatorname{det} B(\lambda)| d \lambda .
$$


Let $\left\{P_{\lambda}: \lambda \in \Sigma\right\}$ be a field of projections defined on $L^{2}\left(\mathbb{R}^{d}\right)$. We say that a left-invariant closed Hilbert subspace of $L^{2}(N)$ is a multiplicity-free subspace if and only if under the Plancherel transform, the Hilbert space corresponds to

$$
\int_{\Sigma}^{\oplus}\left(L^{2}\left(\mathbb{R}^{d}\right) \otimes P_{\lambda}\left(L^{2}\left(\mathbb{R}^{d}\right)\right)\right)|\operatorname{det} B(\lambda)| d \lambda,
$$

and for almost every $\lambda \in \Sigma$, $\operatorname{rank}\left(P_{\lambda}\right)=1$. Similarly, we say that a left-invariant closed subspace of $L^{2}(N)$ is of finite multiplicity if and only if the image of the Hilbert space under the Plancherel transform is equal to

$$
\int_{\Sigma}^{\oplus}\left(L^{2}\left(\mathbb{R}^{d}\right) \otimes P_{\lambda}\left(L^{2}\left(\mathbb{R}^{d}\right)\right)\right)|\operatorname{det} B(\lambda)| d \lambda,
$$

such that for almost every $\lambda \in \Sigma, \operatorname{rank}\left(P_{\lambda}\right)$ is finite. In this section, we will deal with the existence and construction of Parseval wavelet frames for multiplicity-free, and finite multiplicity closed left-invariant subspaces of $L^{2}(N)$.

We fix $\mathbf{u} \in L^{2}\left(\mathbb{R}^{d}\right)$ such that $\|\mathbf{u}\|_{L^{2}\left(\mathbb{R}^{d}\right)}=1$. Let

$$
\{\mathbf{u}(\lambda)=\mathbf{u}: \lambda \in E\}
$$

be a measurable field of unit vectors in $L^{2}\left(\mathbb{R}^{d}\right)$.

Proposition 17. Let $N$ be a simply connected, connected nilpotent Lie group satisfying $C 1, C 2$, and C3. We fix a Jordan-Hölder basis for $\mathfrak{n}$ such that for a.e. linear functional $\lambda \in E$, we have $|\operatorname{det} B(\lambda)| \leq 1$. There exists a bandlimited function $f \in \mathbf{H}_{E}$, defined as

$$
\begin{aligned}
\mathbf{H}_{E} & =\mathcal{P}^{-1}\left(\int_{\Sigma}^{\oplus} L^{2}\left(\mathbb{R}^{d}\right) \otimes\left(\mathbf{u} \chi_{E}(\lambda)\right)|\operatorname{det} B(\lambda)| d \lambda\right) \\
& =\mathcal{P}^{-1}\left(\int_{E}^{\oplus}\left(L^{2}\left(\mathbb{R}^{d}\right) \otimes \mathbf{u}\right)|\operatorname{det} B(\lambda)| d \lambda\right)
\end{aligned}
$$

such that the system $\{L(\gamma) f: \gamma \in \Gamma\}$ forms a Parseval frame in $\mathbf{H}_{E}$. Moreover, $\|f\|_{\mathbf{H}_{E}}=\mu(E)^{1 / 2} \leq 2^{\frac{2 d-n}{2}}$.

Proof. For every fixed linear functional $\lambda \in E$ (see 11) since $|\operatorname{det} B(\lambda)|$ is less or equal to one, there exists a function $\phi(\lambda) \in L^{2}\left(\mathbb{R}^{d}\right)$ such that the Gabor system $\mathcal{G}\left(\phi(\lambda), \mathbb{Z}^{2} \times \operatorname{det} B(\lambda) \mathbb{Z}^{2}\right)$ forms a Parseval frame a.e. This is due to the density condition given in Proposition 1. Now, let us define a function $f \in \mathbf{H}_{E}$ such that

$$
\mathcal{F} f(\lambda)=\left(|\operatorname{det} B(\lambda)|^{-1 / 2} \phi(\lambda) \otimes \mathbf{u}\right) \chi_{E}(\lambda) .
$$


Referring to (5), we recall that

$$
\mathcal{F}(L(\gamma) f)(\lambda)=\pi_{\lambda}(\gamma) \circ \mathcal{F} f(\lambda)=|\operatorname{det} B(\lambda)|^{-1 / 2}\left(\left(\pi_{\lambda}(\gamma) \phi(\lambda)\right) \otimes \mathbf{u}\right) \chi_{E}(\lambda) .
$$

Let $g$ be any function in $\mathbf{H}_{E}$ such that $\mathcal{F} g(\lambda)=u_{g}(\lambda) \otimes\left(\mathbf{u} \chi_{E}(\lambda)\right)$, and $r(\lambda)=|\operatorname{det} B(\lambda)|^{1 / 2}$. We have

$$
\sum_{\gamma \in \Gamma}\left|\langle g, L(\gamma) f\rangle_{\mathbf{H}_{E}}\right|^{2}=\sum_{\gamma \in \Gamma}\left|\int_{E}\left\langle u_{g}(\lambda), r(\lambda) \pi_{\lambda}(\gamma) \phi(\lambda)\right\rangle_{L^{2}\left(\mathbb{R}^{d}\right)} d \lambda\right|^{2}
$$

which is equal to

$$
\sum_{\eta \in \mathbb{Z}^{2 d}} \sum_{k \in \mathbb{Z}^{n-2 d}}\left|\int_{E} e^{2 \pi i\langle k, \lambda\rangle}\left\langle u_{g}(\lambda), r(\lambda) \pi_{\lambda}(\eta) \phi(\lambda)\right\rangle_{L^{2}\left(\mathbb{R}^{d}\right)} d \lambda\right|^{2} .
$$

Since $\left\{e^{2 \pi i\langle k, \lambda\rangle} \chi_{E}(\lambda)\right\}_{k \in \mathbb{Z}}$ defines a Parseval frame in $L^{2}(E)$, letting

$$
c_{\eta}(\lambda)=\left\langle u_{g}(\lambda),|\operatorname{det} B(\lambda)|^{1 / 2} \pi_{\lambda}(\eta) \phi(\lambda)\right\rangle_{L^{2}\left(\mathbb{R}^{d}\right)},
$$

we obtain

$$
\sum_{\eta \in \Gamma_{2} \Gamma_{3}} \sum_{k \in \mathbb{Z}^{n-2 d}}\left|\int_{E} e^{2 \pi i\langle k, \lambda\rangle} c_{\eta}(\lambda) d \lambda\right|^{2}=\sum_{\eta \in \Gamma_{2} \Gamma_{3}} \sum_{k \in \mathbb{Z}^{n-2 d}}\left|\widehat{c}_{\eta}(k)\right|^{2}
$$

The above equality is simply $\sum_{\eta \in \Gamma_{2} \Gamma_{3}}\left\|c_{\eta}\right\|_{L^{2}(E)}^{2}$. Using the fact that

$$
\mathcal{G}\left(\phi(\lambda), \mathbb{Z}^{2} \times \operatorname{det} B(\lambda) \mathbb{Z}^{2}\right)
$$

is a Parseval frame for almost every $\lambda \in \Sigma$, we obtain $\sum_{\gamma \in \Gamma}\left|\langle g, L(\gamma) f\rangle_{\mathbf{H}_{E}}\right|^{2}$ is equal to

$$
\begin{aligned}
& \int_{E} \sum_{\eta \in \Gamma_{2} \Gamma_{3}}\left|\left\langle u_{g}(\lambda),|\operatorname{det} B(\lambda)|^{1 / 2} \pi_{\lambda}(\eta) \phi(\lambda)\right\rangle_{L^{2}\left(\mathbb{R}^{d}\right)}\right|^{2} d \lambda \\
& =\int_{E} \sum_{\eta \in \Gamma_{2} \Gamma_{3}}\left|\left\langle u_{g}(\lambda), \pi_{\lambda}(\eta) \phi(\lambda)\right\rangle_{L^{2}\left(\mathbb{R}^{d}\right)}\right|^{2}|\operatorname{det} B(\lambda)| d \lambda \\
& =\int_{E}\|\mathcal{F} g(\lambda)\|_{\mathcal{H} \mathcal{S}}^{2}|\operatorname{det} B(\lambda)| d \lambda \\
& =\|g\|_{\mathbf{H}_{E}}^{2} .
\end{aligned}
$$


Now, computing the norm of $f$, we apply the results from Proposition 2, and we obtain

$$
\|f\|_{\mathbf{H}_{E}}^{2}=\int_{E}\|\phi(\lambda)\|_{\mathcal{H} \mathcal{S}}^{2} d \lambda=\int_{E}|\operatorname{det} B(\lambda)| d \lambda=\mu(E) .
$$

Finally, for the last part, since $E \subset\{\lambda \in \Sigma:|\operatorname{det} B(\lambda)| \leq 1\}$, then

$$
\|f\|_{\mathbf{H}_{E}}^{2}=\int_{E}|\operatorname{det} B(\lambda)| d \lambda \leq \int_{E} d \lambda \leq \frac{1}{2^{n-2 d}} .
$$

This concludes the proof.

We would like to remark that the Hilbert space $\mathbf{H}_{E}$ is natually identified with the Hilbert space

$$
L^{2}\left(E \times \mathbb{R}^{d},|\operatorname{det} B(\lambda)| d \lambda d t\right) .
$$

However, it is much more convenient to use the notation of direct integral. We recall that given $\gamma \in \Gamma$,

$$
A(\gamma)=\exp \left(\sum_{k=1}^{n-2 d} 2 m_{k} Z_{k}\right) \exp \left(\sum_{k=1}^{d} 2 n_{k} Y_{k}\right) \exp \left(\sum_{k=1}^{d} j_{k} X_{k}\right) .
$$

Definition 18. (Dilation action) Let $\mathcal{U}\left(L^{2}(N)\right)$ be the group of unitary operators acting in $L^{2}(N)$. We define a unitary representation of the group $H=\left\{A^{j}: j \in \mathbb{Z}\right\}$ acting in $L^{2}(N)$ as follows. $D: H \rightarrow \mathcal{U}\left(L^{2}(N)\right)$ and

$$
\left(D_{A^{j}} f\right)(x)=\operatorname{det}\left(A d_{A}\right)^{-j / 2} f\left(A^{-j}(x)\right)=2^{-j\left(\frac{n-d}{2}\right)} f\left(A^{-j}(x)\right) .
$$

Lemma 19. Given any function $f \in L^{2}(N)$,

$$
\mathcal{F}\left(D_{A^{j}} f\right)(\lambda)=2^{j\left(\frac{n-d}{2}\right)} \mathcal{F} f\left(2^{j} \mathbf{I}_{n-2 d} \lambda\right) .
$$

Proof. Given any $u, v \in L^{2}\left(\mathbb{R}^{d}\right)$,

$$
\begin{aligned}
\left\langle\mathcal{F}\left(D_{A^{j}} f\right)(\lambda) u, v\right\rangle & =\int_{N} D_{A^{j}} f(x)\left\langle\pi_{\lambda}(x) u, v\right\rangle d x \\
& =2^{j\left(\frac{n-d}{2}\right)} \int_{N} f(x)\left\langle\pi_{\lambda}\left(A^{j}(x)\right) u, v\right\rangle d x .
\end{aligned}
$$


For each $\lambda \in \Sigma$, we recall that we identify $\lambda=\left(\lambda_{1}, \cdots, \lambda_{n-2 d}, 0, \cdots, 0\right)$ with $\left(\lambda_{1}, \cdots, \lambda_{n-2 d}\right)$. Also for every $\gamma \in \Gamma$, we have

$$
\pi_{\lambda}\left(A^{j}(\gamma)\right)=\pi_{A^{-j \cdot \lambda}}(\gamma)=\pi_{2^{j} \mathbf{I}_{n-2 d} \lambda}(\gamma)
$$

where $A^{-j} \cdot \lambda$ denotes the coadjoint action of $A^{-j}$ on a linear functional $\lambda$. Since

$$
\left\langle\mathcal{F}\left(D_{A^{j}} f\right)(\lambda) u, v\right\rangle=2^{j\left(\frac{n-d}{2}\right)} \int_{N} f(x)\left\langle\pi_{2^{j} \mathbf{I}_{n-2 d} \lambda}(\gamma) u, v\right\rangle d x,
$$

is true for all $u, v \in L^{2}\left(\mathbb{R}^{d}\right)$, we obtain

$$
\mathcal{F}\left(D_{A^{j}} f\right)(\lambda)=2^{j\left(\frac{n-d}{2}\right)} \mathcal{F} f\left(2^{j} \mathbf{I}_{n-2 d} \lambda\right) .
$$

Definition 20. Given $j \in \mathbb{Z}$, we define the Hilbert space

$$
\mathbf{H}_{E}^{j}=\mathcal{P}^{-1}\left(\int_{2^{-j} \mathbf{I}_{n-2 d} E}^{\oplus}\left(L^{2}\left(\mathbb{R}^{d}\right) \otimes \mathbf{u}\right)|\operatorname{det} B(\lambda)| d \lambda\right) .
$$

The choice of dilation induced by the action of $A$ has been carefully chosen in Corollary 10 so that the following lemma is indeed possible. We would like to notice that for general dilations, Lemma 21 below is false. That is the dilation coming from $A$ is a special type of dilation.

Lemma 21. For any $j \in \mathbb{Z}$,

$$
\mathcal{P}\left(D_{A^{j}}\left(\mathbf{H}_{E}\right)\right) \subset \int_{2^{-j} \mathbf{I}_{n-2 d} E}^{\oplus}\left(L^{2}\left(\mathbb{R}^{d}\right) \otimes \mathbf{u}\right)|\operatorname{det} B(\lambda)| d \lambda .
$$

Proof. Let $f \in \mathbf{H}_{E}$. For almost every $\lambda \in E$, there exists $\phi(\lambda) \in L^{2}\left(\mathbb{R}^{d}\right)$ such that $\mathcal{F} f(\lambda)=\phi(\lambda) \otimes\left(\mathbf{u}\left(\chi_{E}(\lambda)\right)\right)$. Next we have

$$
\mathcal{F}\left(D_{A^{j}} f\right)(\lambda)=2^{j\left(\frac{n-d}{2}\right)} \phi\left(2^{j} \mathbf{I}_{n-2 d} \lambda\right) \otimes\left(\mathbf{u}\left(\chi_{E}\left(2^{j} \mathbf{I}_{n-2 d} \lambda\right)\right)\right) .
$$

Proposition 22. For $j, j^{\prime} \in \mathbb{Z}$, the following hold.

1. $L\left(A^{j} \gamma\right) D_{A^{j}}=D_{A^{j}} L(\gamma)$. 
2. Let $f$ be such that $L(\Gamma) f$ is a Parseval frame in $\mathbf{H}_{E}$. The system

$$
\left\{L\left(A^{j} \gamma\right) D_{A^{j}} f: \gamma \in \Gamma\right\}
$$

forms a Parseval frame for $\mathbf{H}_{E}^{j}$.

3. $\left(D_{A^{j}} L(\Gamma)\right)\left(\mathbf{H}_{E}\right)=\mathbf{H}_{E}^{j}$.

4. For $j \neq j^{\prime}, \mathbf{H}_{E}^{j} \perp \mathbf{H}_{E}^{j^{\prime}}$.

5. Let $\Sigma$ be the support of the Plancherel measure of the group $N$ as defined in (4).

$$
\bigoplus_{j \in \mathbb{Z}} \mathbf{H}_{E}^{j}=\mathcal{P}^{-1}\left(\int_{\Sigma}^{\oplus}\left(L^{2}\left(\mathbb{R}^{d}\right) \otimes \mathbf{u}\right)|\operatorname{det} B(\lambda)| d \lambda\right)
$$

Proof. To prove (1), let $\phi \in L^{2}(N)$, and $\phi_{j}=D_{A^{j}} \phi$. Thus,

$$
L\left(A^{j}(\gamma)\right) D_{A^{j}} \phi(x)=D_{A^{j}} L(\gamma) \phi(x) .
$$

To prove (2) we first show that the sequence

$$
\left\{L\left(A^{j}(\gamma)\right) D_{A^{j}} f: \gamma \in \Gamma\right\}
$$

is total in $\mathbf{H}_{E}^{j}$. Let $g$ be a non zero function in $\mathbf{H}_{E}^{j}$ such that $g$ is orthogonal to the closure of the span of $\left\{L\left(A^{j}(\gamma)\right) D_{A^{j}} f: \gamma \in \Gamma\right\}$. In other words, for all $\gamma \in \Gamma,\left\langle g, L\left(A^{j}(\gamma)\right) D_{A^{j}} f\right\rangle=0$. However,

$$
\begin{aligned}
\left\langle g, L\left(A^{j}(\gamma)\right) D_{A^{j}} f\right\rangle & =\left\langle g, D_{A^{j}} L(\gamma) f\right\rangle \\
& =\left\langle D_{A^{-j}} g, L(\gamma) f\right\rangle .
\end{aligned}
$$

Since $D_{A^{-j}} g \in \mathbf{H}_{E}$, then $D_{A^{-j}} g=0$ and $g=0$. That would be a contradiction. Now, we show that $\left\{L\left(A^{j}(\gamma)\right) D_{A^{j}} f: \gamma \in \Gamma\right\}$ is a frame. Let $g$ be an arbitrary element of $\mathbf{H}_{E}^{j}$. We have

$$
\begin{aligned}
\sum_{\gamma \in \Gamma}\left|\left\langle g, L\left(A^{j}(\gamma)\right) D_{A^{j}} f\right\rangle\right|^{2} & =\sum_{\gamma \in \Gamma}\left|\left\langle g, D_{A^{j}} L(\gamma) f\right\rangle\right|^{2} \\
& =\left\|D_{A^{-j}} g\right\|_{\mathbf{H}_{E}}^{2} \\
& =\|g\|_{\mathbf{H}_{E}^{j}}^{2} .
\end{aligned}
$$

Part (3) is just a direct consequence of Parts (1), and (2), Part (4) is obvious by the definition given in (12). To prove Part (5), we use the fact that the collection of sets $\left\{A^{-j} E: j \in \mathbb{Z}\right\}$ forms a measurable partition of $\Sigma$ and we apply Part (1),(2),(3) and (4). 
Theorem 23. Let $f$ be defined such that $\{L(\gamma) f: \gamma \in \Gamma\}$ is a Parseval frame for $\mathbf{H}_{E}$. The system $\left\{D_{A^{j}} L(\gamma) f: \gamma \in \Gamma, j \in \mathbb{Z}\right\}$ is a Parseval frame for the multiplicity-free space

$$
\bigoplus_{j \in \mathbb{Z}} \mathbf{H}_{E}^{j}=\mathcal{P}^{-1}\left(\int_{\Sigma}^{\oplus}\left(L^{2}\left(\mathbb{R}^{d}\right) \otimes \mathbf{u}\right)|\operatorname{det} B(\lambda)| d \lambda\right) .
$$

Fix an orthonormal basis for $L^{2}\left(\mathbb{R}^{d}\right),\left\{\mathbf{e}_{k}: k \in \mathbb{I}\right\}$ where $\mathbb{I}$ is an infinite countable set. For each $k \in \mathbb{I}$, we obtain the following measurable field of unit vectors $\left\{\mathbf{u}_{k}(\lambda)=\mathbf{e}_{k}: \lambda \in \Sigma\right\}$ and we define

$$
\mathbf{H}_{E}^{j, k}=\mathcal{P}^{-1}\left(\int_{2^{-j} \mathbf{I}_{n-2 d} E}^{\oplus}\left(L^{2}\left(\mathbb{R}^{d}\right) \otimes \mathbf{e}_{k}\right)|\operatorname{det} B(\lambda)| d \lambda\right) .
$$

It is clear that $L^{2}(N)=\bigoplus_{(k, j) \in \mathbb{I} \times \mathbb{Z}} \mathbf{H}_{E}^{j, k}$.

Theorem 24. Let $S$ be a finite subset of $\mathbb{I}$. For $k \in S$, we define $f_{k}$ such that $\left\{L(\gamma) f_{k}: \gamma \in \Gamma\right\}$ is a Parseval frame for $\mathbf{H}_{E}^{0, k}$. The system

$$
\left\{D_{A^{j}} L(\gamma) f_{k}: \gamma \in \Gamma, j \in \mathbb{Z}, k \in S\right\}
$$

is a Parseval frame for

$$
\bigoplus_{(k, j) \in S \times \mathbb{Z}} \mathbf{H}_{E}^{j, k} .
$$

Furthermore, for $k \in \mathbb{I}$, we define $f_{k}$ such that $\left\{L(\gamma) f_{k}: \gamma \in \Gamma\right\}$ is a Parseval frame for $\mathbf{H}_{E}^{0, k}$. The system

$$
\left\{D_{A^{j}} L(\gamma) f_{k}: \gamma \in \Gamma, j \in \mathbb{Z}, k \in \mathbb{I}\right\}
$$

is a Parseval frame for $L^{2}(N)$.

Example 25. A 9-dimensional case.

Let $N$ a be Lie group with Lie algebra $\mathfrak{n}$ spanned by the basis

$$
\left\{Z_{1}, Z_{2}, Z_{3}, Y_{1}, Y_{2}, Y_{3}, X_{1}, X_{2}, X_{3}\right\}
$$

with the following non-trivial Lie brackets.

$$
\begin{aligned}
& {\left[X_{1}, Y_{1}\right]=Z_{1},\left[X_{2}, Y_{1}\right]=Z_{2},\left[X_{3}, Y_{1}\right]=Z_{3},} \\
& {\left[X_{1}, Y_{2}\right]=Z_{2},\left[X_{2}, Y_{2}\right]=Z_{3},\left[X_{3}, Y_{2}\right]=Z_{1}}
\end{aligned}
$$




$$
\left[X_{1}, Y_{3}\right]=Z_{3},\left[X_{2}, Y_{3}\right]=Z_{1},\left[X_{3}, Y_{3}\right]=Z_{2} .
$$

Let $A=\exp U$ such that for all $1 \leq i \leq 3,\left[U, Z_{i}\right]=\ln (2) Z_{i}$ and $\left[U, Y_{i}\right]=$ $\ln (2) Y_{i}$. The Plancherel measure is

$$
d \mu(\lambda)=\left|-\lambda_{1}^{3}+3 \lambda_{1} \lambda_{2} \lambda_{3}-\lambda_{2}^{3}-\lambda_{3}^{3}\right| d \lambda_{1} d \lambda_{2} d \lambda_{3}
$$

and is supported on the manifold

$$
\Sigma=\left\{\left(\lambda_{1}, \lambda_{2}, \lambda_{3}, 0, \cdots, 0\right) \in \mathfrak{n}^{*}:-\lambda_{1}^{3}+3 \lambda_{1} \lambda_{2} \lambda_{3}-\lambda_{2}^{3}-\lambda_{3}^{3} \neq 0\right\}
$$

which we identify with a Zariski open subset of $\mathbb{R}^{3}$. Let

$$
E=\left([-1 / 2,1 / 2)^{3} \backslash[-1 / 4,1 / 4)^{3}\right) \cap \Sigma .
$$

For each $\lambda \in E$, there exists a Gabor system $\mathcal{G}\left(g(\lambda), \mathbb{Z}^{3} \times B(\lambda) \mathbb{Z}^{3}\right)$ which is a Parseval frame in $L^{2}\left(\mathbb{R}^{3}\right)$ for

$$
B(\lambda)=\left(\begin{array}{lll}
\lambda_{1} & \lambda_{2} & \lambda_{3} \\
\lambda_{2} & \lambda_{3} & \lambda_{1} \\
\lambda_{3} & \lambda_{1} & \lambda_{2}
\end{array}\right) .
$$

We define $f \in L^{2}(N)$ such that

$$
\mathcal{F} f(\lambda)=\left(\left|-\lambda_{1}^{3}+3 \lambda_{1} \lambda_{2} \lambda_{3}-\lambda_{2}^{3}-\lambda_{3}^{3}\right|^{-1 / 2} g(\lambda) \otimes \chi_{[0,1]^{3}}\right) \chi_{E}(\lambda) .
$$

The system $\left\{D_{A^{j}} L(\gamma) f: \gamma \in \Gamma, j \in \mathbb{Z}\right\}$ forms a Parseval frame in

$$
\mathcal{P}^{-1}\left(\int_{\Sigma}^{\oplus} L^{2}\left(\mathbb{R}^{3}\right) \otimes \chi_{[0,1]^{3}} d \mu(\lambda)\right),
$$

and $\|f\|_{L^{2}(N)}^{2}=7.76 \times 10^{-2}$. Let

$$
\left\{\beta_{n, k}(t)=\exp (2 \pi i\langle t, n\rangle) \chi_{[0,1]^{3}}(t-k): n \in \mathbb{Z}^{3}, k \in \mathbb{Z}^{3}\right\}
$$

be an orthonormal basis in $L^{2}\left(\mathbb{R}^{3}\right)$. We define

$$
\mathcal{F} f_{n, k}(\lambda)=\left(\left|-\lambda_{1}^{3}+3 \lambda_{1} \lambda_{2} \lambda_{3}-\lambda_{2}^{3}-\lambda_{3}^{3}\right|^{-1 / 2} g(\lambda) \otimes \beta_{n, k}\right) \chi_{E}(\lambda) .
$$

The system

$$
\left\{D_{A^{j}} L(\gamma) f_{n, k}: \gamma \in \Gamma, j \in \mathbb{Z}, \quad(n, k) \in \mathbb{Z}^{3} \times \mathbb{Z}^{3}\right\}
$$

forms a Parseval frame in $L^{2}(N)$. 
Remark 26. The tools used for the construction of Parseval frames in this paper are available to us because this class of nilpotent Lie group admits unitary irreducible representations which behave quite well. For other type of nilpotent Lie groups, it is not clear how to generalize our construction. Here is a fairly simple example where we encounter some major obstructions. Let $N$ be a nilpotent Lie group with Lie algebra $\mathfrak{n}$ over the reals, spanned by $\{Z, Y, X, W\}$ with the following non-trivial Lie brackets

$$
[X, Y]=Z,[W, X]=Y .
$$

$N$ is a step-3 nilpotent Lie group, and its dual is parametrized by a Zariski open subset of $\mathbb{R}^{2}$. However, notice that the center of this group is only onedimensional, and the group itself is not square-integrable mod the center. The construction provided in this paper does not seem to work even for such a simple group. The main obstruction is related to the fact that the irreducible representations corresponding to elements in $N / Z(N)$ do not yield to a Gabor system. There are even step-two nilpotent Lie groups for which our method does not work. For example, let $N$ be a freely-generated step-two nilpotent Lie group with 3 generators $Z_{1}, Z_{2}, Z_{3}$, with non-trivial Lie brackets

$$
\left[Z_{1}, Z_{2}\right]=Z_{12},\left[Z_{1}, Z_{3}\right]=Z_{13} \text {, and }\left[Z_{2}, Z_{3}\right]=Z_{23} \text {. }
$$

It remains unclear at this point if it is possible to construct Parseval frames using similar techniques.

\section{References}

[1] L. Corwin, L. Greenleaf, Representations of nilpotent Lie groups and their applications. Part I. Basic theory and examples. Cambridge Studies in Advanced Mathematics, 18. Cambridge University Press, Cambridge, 1990.

[2] B. Currey, A. Mayeli; Gabor fields and wavelet sets for the Heisenberg group, Monatsh. Math. 162, No. 2, 119-142 (2011)

[3] A. Mayeli, Discrete and continuous wavelet transformation on the Heisenberg group, Ph.D thesis, 2005.

[4] B. Currey, Admissibility for a class of quasiregular representations. Canad. J. Math. 59 (2007), no. 5, 917-942. 
[5] B. Currey, T. McNamara, Decomposition and admissibility for the quasiregular representation for generalized oscillator groups. Radon transforms, geometry, and wavelets, 51-73, Contemp. Math., 464, Amer. Math. Soc., Providence, RI, 2008.

[6] X. Dai, D. Larson, D. Speegle, Wavelet sets in Rn. J. Fourier Anal. Appl. 3 (1997), no. 4, 451-456

[7] D. Han and Y. Wang. Lattice tiling and the Weyl-Heisenberg frames. Geom. Funct. Anal., 11(4):742-758, 2001.

[8] E. Hernandez, G. Weiss, A First Course in Wavelets, CRC Press, Boca Raton (1996).

[9] H. Führ, Abstract harmonic analysis of continuous wavelet transforms, Lecture Notes in Mathematics 1863. Berlin: Springer.

[10] H. Liu, L. Peng, Admissible wavelets associated with the Heisenberg group, Pacific Journal of Mathematics, 180 (1997), 101-123.

[11] P. Lemarié, Base d'ondelettes sur les groupes de Lie stratifiés. (French) [Basis of wavelets on stratified Lie groups] Bull. Soc. Math. France 117 (1989), no. 2, 211-232.

[12] P. Lemarié, Wavelets, spline interpolation and Lie groups, Harmonic Analysis (Sendai, 1990), Springer, Tokyo, 1991, 154-164.

[13] V. Oussa, Bandlimited Spaces on Some 2-step Nilpotent Lie Groups With One Parseval Frame Generator, to appear in Rocky Mountain Journal of Mathematics. 